\title{
Células Neuroendocrinas en Yemas Gustativas Primordiales
}

\author{
Neuroendocrine Cells in Primordial Taste Buds
}

\author{
Ricardo Fierro-Murga*; Alejandro Ceballos-Salobreña*** \& Luis Gaitán-Cepeda***
}

\begin{abstract}
FIERRO-MURGA, R.; CEBALLOS-SALOBREÑA, A. \& GAITÁN-CEPEDA, L. Células neuroendocrinas gustativas primordiales. Int. J. Morphol., 31(2):444-448, 2013.

RESUMEN: El objetivo del trabajo fue identificar la presencia de células neuroendócrinas en yemas gustativas primordiales (fetales) humanas. Fueron utilizadas 15 lenguas fetales humanas obtenidas de abortos espontáneos (tiempo de gestación $\leq 23$ semanas) de pacientes ingresados en el Hospital General, Chihuahua, México. Una muestra representativa del ápex lingual fue embebida en parafina y cortada a 3 micras para ser procesadas con la técnica inmunohitoquímica utilizando los siguientes anticuerpos: Anti-sinaptofisina; anti-proteína neurofilamento; anti-cromogranina; anti-citoqueratina 20; y anti proteínas-S100. El protocolo de investigación fue aprobado por los comités de bioética de las instituciones participantes. Se obtuvo la autorización de los padres para la utilización del material biológico. Las células gustativas fetales fueron fuertemente positivas a cromogranina, y negativas a sinaptofisina. Las papilas gustativas fetales humanas mostraron inmunorreactividad positiva contra citoqueratina 20 , mientras que fibras nerviosas intragemulares mostraron inmunorreactividad contra anticuerpos anti-proteína neurofilamento. Las fibras nerviosas subyacentes al epitelio gustativo fueron positivas a proteína S100. Se muestra evidencia inmunohistoquímica de la presencia de células neuroendócrinas gustativa en yemas gustativas linguales primordiales humanas. Esto sugiere una probable participación neuroendocrina o paracrina en el desarrollo de las yemas gustativas humanas.
\end{abstract}

PALABRAS CLAVE: Yemas gustativas; Células gustativas; Neuroendócrino; Cromogranina.

\section{INTRODUCCIÓN}

Las yemas gustativas son receptores epiteliales altamente especializados cuya citoarquitectura ha sido bien descrita (Just et al., 2005; Miura et al., 2006). Sin embargo, los procesos que intervienen en su desarrollo y maduración no son totalmente comprendidos. Se han propuesto dos modelos de participación neural en el desarrollo de las yemas gustativas. El modelo de "inducción neural" propone que fibras nerviosas periféricas inducen la formación de las yemas gustativas; mientras que el modelo de "especificación temprana" sugiere la existencia de áreas específicas en el epitelio orofaríngeo embrionario que son inducidas hacia epitelio gustativo durante la gastrulación. Posteriormente las yemas gustativas se desarrollarán en este epitelio especificado a través de inducción paracrina (Northcutt, 2004). Sin embargo y a pesar del neurotrofismo mostrado por las yemas gustativas (Farbam, 1972), modelos experimentales han podido demostrar la formación de yemas gustativas aún en ausencia de contacto con tejido neural (Barlow et al., 1996; Graziadei \& DeHan, 1973; Toyoshima et al., 1984).

Recientemente Itoa y colaboradores (Itoa et al., 2010), utilizando como modelo experimental ratones BDNFxNT-3 double knockout demostraron que la formación de células gustativas no requiere la presencia de inervación gustativa y somatosensorial (etapa independiente del nervio). Sin embargo, las células gustativas requieren la presencia de inervación para su mantenimiento y maduración (fase dependiente del nervio) (Itoa et al.). Estos hallazgos sugieren un posible participación paracrina o neuroendocrina en la formación de las yemas gustativas. Sin embargo, en nuestro conocimiento, la presencia de células neuroendocrinas en yemas gustativas en desarrollo no ha sido demostrada. Por lo anterior el principal objetivo del presente trabajo fue la identificación inmunohistoquímica de células neuroendocrinas en yemas gustativas humanas fetales.

\section{MATERIAL Y MÉTODO}

Quince lenguas fetales humanas fueron incluidas en el presente estudio. Los fetos fueron secuelas de abortos espontáneos de pacientes atendidos en la unidad ginecológica en el Hospital General, Chihuahua, México. Ninguno de los fetos

* Escuela de Medicina, Universidad Autónoma de Chihuahua, Chihuahua, México.

** Departamento de Medicina Oral, Facultad de Odontología, Universidad de Granada, Granada, España.

**** Laboratorio de Patología Clínica y Experimental, Facultad de Odontología, Universidad Nacional Autónoma de México, México. 
mostró, macroscópicamente, dismorfologías. La edad gestacional de los fetos fue establecida siguiendo los criterios propuestos por Lewis y Perrin (Lewis \& Perrin, 1999). De acuerdo con estos criterios, el promedio de la longitud céfalo - pélvica, de los fetos fue $18,1 \mathrm{~cm} \pm 2,45 \mathrm{~cm}$ (rango 19,5-16,3 $\mathrm{cm})$. Para propósitos de investigación las muestras fueron agrupadas según su edad gestacional en dos grupos: Grupo 1, cuyas edades gestacionales fueron $\leq 18$ semanas postovulación, formado por 8 fetos; y grupo 2 compuesto por 7 fetos cuyas edades gestacionales fueron $\geq 19$ semanas postovulación.

Las lenguas fueron removidas y una muestra representativa de su ápex fue obtenida y fijada en formalina tamponada al $10 \%$ por un mínimo de 24 horas. Después del tiempo de fijación, las muestras fueron embebidas en parafina, cortadas a $3 \mu \mathrm{m}$ y teñidas con la técnica de tinción de Hematoxilina-Eosina. Cortes histológicos adicionales fueron obtenidos y procesados con la técnica de inmunohistoquímica. La técnica de inmunohistoquímica fue realizada con el kit Immunodetector HRP/DAB (BioSB ®, Santa Bárbara, CA). Posterior a su incubación en anticuerpos primarios, las muestras fueron lavadas e incubadas en los anticuerpos secundarios siguientes: anti-sinaptofisina (dilución 1:1500), anti-proteína neurofilamento (PNF) (dilución 1:100), anticromogranina (dilución 1:300), anti-citoqueratina 20 (CK20) (dilución 1:50) y anti proteína-S100 (dilución 1:800). Los anticuerpos anti-sinaptofisina y anti-cromogranina fueron escogidos para identificar células gustativas neuroendocrinas.

El anticuerpo anti-cromogranina es un marcador inmunohistoquímico específico de células neuroendócrinas (Jensen et al., 1990; Leone \& Taccagni, 1990; Wiedenmann et al., 1988). Los anticuerpos anti-PNF y anti proteina-S100 fueron utilizados para identificar fibras nerviosas. El anticuer- po anti-CK20 fue seleccionado al ser considerado un inmunomarcador específico de yemas gustativas (Barrett $e t$ al., 2000). El control positivo para anti-sinaptofisina fue tejido cerebral; para anti-cromogranina y anti-CK20 fue tejido pancreático. El control positivo a proteína S100 fue un neurilemoma y para anti-PNF fue tejido nervioso periférico. Las muestras fueron codificas de tal manera que los observadores (RFG/ACS) nunca conocieron la edad fetal de cada muestra. El protocolo de investigación fue aprobado por los comités de bioética de las instituciones involucradas. El servicio de patología, así como los padres otorgaron su autorización para el uso del material biológico.

\section{RESULTADOS}

Cromogranina. De las ocho muestras del grupo 1, cinco $(62,5 \%$ presentaron una intensa reacción positiva a cromogranina en el epitelio gustativo. De las siete muestras del grupo 2, seis $(85.7 \%)$ mostraron inmunoreacción positiva a la cromogranina en las células gustativas de yemas gustativas desarrolladas (Fig. 1-A). Las células gustativas cromogranina-positivas tuvieron una forma ahusada con una prolongación citoplasmática dirigida hacia la región de poro gustativo (Fig. 1-B).

Citoqueratina 20. El epitelio gustativo del grupo 1 fue levemente positivo a CK20 (Fig. 1-C), mientras que en el grupo 2 el epitelio gustativo y las yemas gustativas fueron fuertemente positivos a este mismo anticuerpo (Fig. 1-D).

Proteína S100. En ambos grupos, las fibras nerviosas situadas debajo de las papilas fungiformes mostraron fuerte

Tabla I. Características inmunohistoquímicas de yemas gustativas primordiales humanas.

\begin{tabular}{|c|c|c|c|c|c|c|}
\hline & $\begin{array}{l}\text { Caso n ùmero (edad } \\
\text { gestacional en semanas) }\end{array}$ & Ck 20 & S-100 & PNF & CROMGR & SINPT \\
\hline \multirow{8}{*}{$\begin{array}{l}\text { Grupo } 1 \text { ( } \text { Edad } \\
\text { postovulatoria } \leq 18 \\
\text { semanas) }\end{array}$} & $1(14)$ & + & - & - & + & - \\
\hline & 2 (16) & - & - & - & - & - \\
\hline & 3 (16) & + & - & + & + & - \\
\hline & 4 (16) & + & - & - & - & - \\
\hline & $5(16)$ & + & - & -- & + & - \\
\hline & $6(16)$ & + & - & - & + & - \\
\hline & 7 (18) & - & - & - & - & - \\
\hline & 8 (18) & + & - & - & + & - \\
\hline \multirow{7}{*}{$\begin{array}{l}\text { Grupo } 2 \quad \text { (Edad } \\
\text { postovulatoria } \geq 19 \\
\text { semanas) }\end{array}$} & 9 (19) & + & - & + & + & - \\
\hline & 10 (19) & + & - & - & - & - \\
\hline & 11 (19) & + & - & - & + & - \\
\hline & $12(20)$ & + & - & + & - & - \\
\hline & $13(21)$ & + & - & - & + & - \\
\hline & $14(22)$ & + & - & + & + & - \\
\hline & $15(22)$ & + & - & + & + & - \\
\hline
\end{tabular}



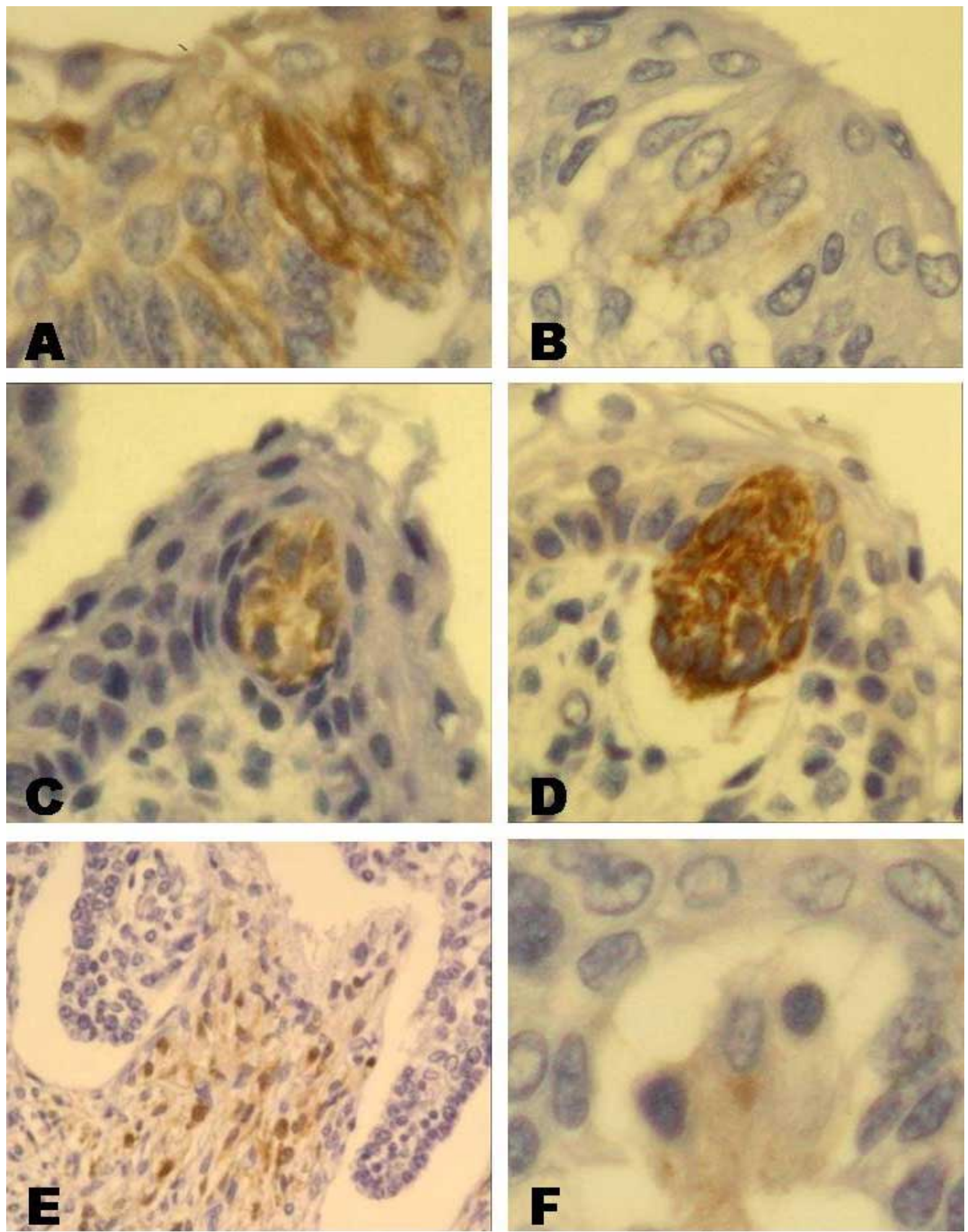

Fig. 1. Características inmunohistoquímicas de yemas gustativas primordiales humanas. A. Células gustativas de yemas gustativas ptimordiales de un feto humano, mostrando fuerte inmunoreactividad a cromogranina. Grupo 1. X1000. B.- Célula gustativa inmunopositiva a cromogranina. Se observa su forma ahusada. Grupo 2. X1000. C. Yema gustativa mostrando inmunoreactividad a citoqueratina 20. Grupo 2. X400. D. Células gustativas con fuerte inmunoreactividad a citoqueratina 20. Grupo 1. X1000.E. Fibra nerviosa localizada en el core de una papilla lingual con inmunoreacción a proteína S-100. Grupo 2. X250. F. Esta microfotografía muestra fibras nerviosas intragemulares con inmunoreacción a pretína del neurofilamento. Grupo 1. x400. 
inmunorreacción a proteína S100 (Fig. 1-E). No se observó inmunorreactividad a la proteína $\mathrm{S} 100$ en los tejidos epiteliales, ya fueran gustativos o no gustativos.

Proteína del neurofilamento. En el grupo 2 tanto las fibras nerviosas libres así como las fibras intragemulares mostraron reacción inmunohistoquímica positiva contra PNF (Fig. $1-\mathrm{F})$.

Sinaptofisina. En ambos grupos no se encontró inmunorreactividad contra sinaptofisina en el epitelio gustativo.

El total de características inmunohistoquímicas de las muestras analizadas son mostradas en la Tabla I.

\section{DISCUSIÓN}

Este artículo proporciona nueva información acerca de las características inmunohistoquímicas de las yemas gustativas humanas, específicamente sobre la presencia de células gustativas neuroendócrinas. Se identificó inmunoreacción positiva a CK20, proteína S100, PNF y, de acuerdo con el principal objetivo, hacia cromogranina. Los resultados sugieren que las células gustativas fueron cromogranina-positivas pero sinaptofisina-negativas.

La presencia de cromogranina en las yemas gustativas ha sido reportado por Dvoryanchikov et al. ( 2007). En células neuroendócrinas, la cromogranina es un componente de las vesículas de centro-denso grande que almacenan monoaminas hasta su eventual liberación. En yemas gustativas desarrolladas de ratones la cromogranina está dispuesta citoplasmáticamente en las células presinápticas (células gustativas tipo III) (Dvoryanchikov et al.). En el presente reporte las células gustativas mostraron fuerte inmunoreactividad a el anticuerpo anti-cromogranina. Este es el primer informe que muestra inmunorreactividad positiva a cromogranina en yemas gustativas humanas en desarrollo.

En el desarrollo de las yemas gustativas ha sido sugerido una probable participación neuroendocrina (Barlow et al.; Graziadei et al.; Toyoshima et al.) y paracrina célulacélula (Asano-Miyoshi et al., 2009). Nuestros resultados apoyan morfológicamente esta sugerencia, sin embargo, es necesario que estos hallazgos sean confirmados. Una doble técnica de tinción inmunoquímica anti-CK20 y anticromogranina pudiera ser útil.

La sinaptofisina es una importante proteína integral de la membrana de las vesículas sinápticas, que pueden es- tar presentes en células no neuronales, por ejemplo, células b pancreáticas y células cromafines (Thomas-Reetz \& De Camilli, 1994). Asano-Miyoshi y colaboradores (AsanoMiyoshi et al.) utilizando muestras de tejido lingual de ratas Wistar de 5 semanas de edad, reportó la presencia de sinaptofisina en yemas gustativas, proponiendo que las células gustativas expresan sinaptofisina como un probable componente de su sistema de neurotransmisión. No observamos inmunorreacción a sinaptofisina en las yemas gustativas o en el epitelio gustativo. Una posible justificación para este hecho pudiera radicar en las diferencias en los diseños experimentales.

El anticuerpo anti-CK20 es un marcador inmunohistoquímico selectivo del epitelio gastrointestinal, urotelio, células de Merkel y algunos carcinomas de células pequeñas de glándulas salivales (Chan et al.); además, y de especial importancia para el presente estudio, se ha reportado que las yemas gustativas muestran inmunorreactividad a este anticuerpo (Barrett et al.). Reportes de la literatura científica sugieren que la inmunorreactividad positiva a CK20 mostrada por las yemas gustativas es posterior a la presencia de fibras nerviosas (Witt et al., 2000).

La proteína S100 y la PNF están relacionadas a diferentes proteínas presentes en fibras nerviosas. Identificamos inmunorreactividad a los anticuerpos anti-proteína S100 y anti-PNF en las fibras nerviosas localizadas en el core de tejido conectivo de las papilas fungiformes. La presencia de fibras nerviosas S100-positivo en papilas fungiformes y en el tejido conectivo subyacente a las yemas gustativas ha sido reportado (Astbäck et al., 1995)

Los resultados del presente estudio muestran que las células gustativas en desarrollo son sinaptofisina-negativas. Esto sugiere una yema gustativa primordial no funcional. Entonces pudiera ser propuesto que la inmunorreactividad contra cromogranina podría estar relacionada con vesículas de núcleo-denso grande que almacenan NE y como tal ser evidencia indirecta de la participación neuroendocrina en el desarrollo de las yemas gustativas. Sin embargo a la fecha esta suposición es meramente especulativa por lo que diseños experimentales y protocolos de investigación deben ser diseñados ex-profesor para rechazar o aceptar esta hipótesis.

En conclusión, los hallazgos del presente reporte ofrecen evidencia inmunohistoquímica de la presencia de células gustativas neuroendocrinas en yemas gustativas fetales humanas La importancia de la participación neuroendocrina en la ontogenia de las yemas gustativas debe establecerse utilizando un proyecto de investigación experimental animal diseñado especialmente para ese propósito. 
FIERRO-MURGA, R.; CEBALlOS-SALOBREÑA, A. \& GAITÁN-CEPEDA, L. Células neuroendocrinas gustativas primordiales. Int. J. Morphol., 31(2):444-448, 2013.

SUMMARY: The objective was to identify the presence of neuroendocrine cells in primary taste buds (fetal) cells. We used 15 human embryionic fetal tongues derived from human spontaneous abortions ( 23 weeks gestation time) of patients admitted to the General Hospital, Chihuahua Mexico. A representative sample of lingual apex was embedded in paraffin and cut to 3 microns processed by immune histochemical technique using the following antibodies: anti-synaptophysin, neuro filament anti-protein, anti chromogranin; anti-citokeratin 20 and S100 anti proteins. Research was approved by bioethics committees of the participating institutions. Permission was obtained from the parents to use the biological material. The taste bud fetal cells were significantly positive for chromogranin and synaptophysin negative. The human fetal taste buds showed positive immunoreactivity against cyto keratin 20 , while nerve fibers underlying the gustatory epithelium were positive for S100 protein. Immunohistochemical evidence shows the presence of neuro endocrine cells in human primordial taste bud papilla. This suggests a probable neuro endocrine or paracrine participation in the development of human taste buds.

KEY WORDS: Taste buds; Taste bud cells; Neuroendocrine; Chromogranin.

\section{REFERENCIAS BIBLIOFRÁFICAS}

Asano-Miyoshi, M.; Hamamichi, R.; Emori, Y. Synaptophysin as a probable component of neurotransmission occurring in taste receptor cells. J Mol. Histol. 2009; 40: 59-70.

Astbäck, J.; Arvidson, K.; Johansson, O. Neurochemical markers of human fungiform papillae and taste buds. Regul. Pept. 1995; 59: 389-98.

Barlow, L.A.; Chien, C.B.; Northcutt, R.G. Embryonic taste buds develop in the absence of innervation. Development 1996; 122: 1103-11.

Barrett, A. W.; Cort, E.M.; Patel, P.; Berkovitz, B. K. An immunohistological study of cytokeratin 20 in human and mammalian oral epithelium. Arch Oral Biol 2000; 45: 879-87.

Chan, J.K.; Suster, S.; Wenig, B.M.; Tsang, W.Y.; Chan, J.B.; Lau, A.L. Cytokeratin 20 immunoreactivity distinguishes Merkel cell (primary cutaneous neuroendocrine) carcinomas and salivary gland small cell carcinomas from small cell carcinomas of various sites. Am. J. Surg. Pathol. 1997; 21: 226-34.

Dvoryanchikov, G.; Tomchik, S.M.; Chaudhari, N. Biogenic amine synthesis and uptake in rodent taste buds. J. Comp. Neurol. 2007; 505: 302-13.

Farbam, A.I. Differentiation of taste buds in organ culture. J. Cell. Biol. 1972; 52: 489-93.

Graziadei, P.; DeHan, R.S. The innervation of frog's taste organ: "a histochemical study". Life Sci. 1973; 13: 1435-49.

Itoa, A.; Nosrata, I.V.; Nosrat, C.A. Taste cells formation does not require gustatory and somatosensory innervations. Neuroscience 2010; 3:18994.

Jensen, S.M.; Gazdar, A.F.; Cuttitta, F.; Russell, E.K.; Linnoila, R.I. A comparison of synaptophysin, chromogranin, and L-dopa decarboxylase as markers for neuroendocrine differentiation in lung cancer cell lines. Cancer Res 1990; 50: 6068-74.

Just, T.; Stave, J.; Pau, H.W.; Guthoff, R. In vivo observation of papillae of the human tongue using confocal laser scanning microscopy. ORL J. Otorhinolaryngol. Relat. Spec. 2005; 67: 207-12.
Leone, B. E.; Taccagni, G.L.; Dell'Antonio, G. \& Cantaboni, A. Chromogranin A as a marker of neuroendocrine histogenesis of tumours: an immunoelectron microscopic study with considerations about the influence of fixation and embedding media on immunolabelling. Basic. Appl. Histochem., 34:143-53, 1990.

Lewis, S.H.; Perrin, E. Pathology of the placenta. $2^{\text {nd }}$ Edi. Saint Louis, Churchill Livingstone, 1999.

Miura, H.; Kusakabe, Y. \& Harada, S. Cell lineage and differentiation in taste buds. Arch. Histol. Cytol., 69: 209-25, 2006.

Northcutt, R. G. Taste buds: development and evolution. Brain Behav. Evol. 2004; 64: 198-206.

Thomas-Reetz, A. C. \& De Camilli, P. A role for synaptic vesicles in non-neuronal cells: clues from pancreatic beta cells and from chromaffin cells. FASEB J., 8:209-16, 1994.

Toyoshima, K.; Honda, E.; Nakahara, S. \& Shimamura, A. Ultrastructural and histochemical changes in the frog taste organ following denervation. Arch. Histol. Jpn., 47: 31-42, 1984.

Wiedenmann, B.; Waldherr, R.; Buhr, H.; Hille, A.; Rosa, P. \& Huttner ,W.B. Identification of gastroenteropancreatic neuroendocrine cells in normal and neoplastic human tissue with antibodies against synaptophysin, chromogranin A, secretogranin I (chromogranin B), and secretogranin II. Gastroenterology, 95:1364-74, 1988.

Witt, M.; Reutter, K.; Ganchrow, D. \& Ganchrow, J. R. Fingerprinting taste buds: intermediate filaments and their implication for taste bud formation. Philos Trans. R. Soc. Lond. B. Biol. Sci., 355:1233-7, 2000.

Dirección para correspondencia:

Dr. Luis Alberto Gaitán Cepeda

Laboratorio de Patología, Facultad de Odontología

Universidad Nacional Autónoma de México

Circuito Institutos s/n, Ciudad Universitaria

Coyoacán 04510, México, D.F.

MÉXICO

Email: Igaitan@unam.mx

Recibido : 04-09-2012

Aceptado: 29-01-2013 